\title{
Spinal Seroma in a Patient with Lumbar Fusion
}

\author{
Ivan Urits, MD', Omar Viswanath, MD $^{2-4}$, Vwaire Orhurhu, MD', Alan D Kaye, MD, $\mathrm{PhD}^{5}$, and \\ Viet Cai, MD ${ }^{1}$
}

Low back pain is a pervasive problem which affects up to $80 \%$ of individuals at least once during their lifetime (1). Unsurprisingly, the rate of patients who opt for surgical fusion as treatment of their low back pain symptoms continues to increase (2). Common indications for lumbar spinal fusion surgery include degenerative disc disease, spondylolisthesis, and spinal stenosis. Though frequently effective for long term management of low back pain symptoms, spinal fusion surgery is associated with various complications which may include persistent or even worsening low back pain. Seroma formation following posterior laminectomy and fusion is a rare though potentially serious complication. While it typically presents as an acute sequala of intraoperative dural tear, spinal seroma can have delayed presentation leading to the progression of significant back pain.

Our patient was a 72-year-old female who had a history of L4 through S1 anterior and posterior spinal fusion performed two years prior uncomplicated with good post-surgical result. Informed consent was obtained for the presentation of this report. She presented to clinic with low back pain and bilateral radicular symptoms that started 6 weeks prior follow-

From : 'Beth Israel Deaconess Medical Center, Department of Anesthesia, Critical Care, and Pain Medicine, Harvard Medical School, Boston, MA; ${ }^{2}$ Valley Anesthesiology and Pain Consultants, Phoenix, AZ; ${ }^{3}$ University of Arizona College of Medicine - Phoenix, Department of Anesthesiology, Phoenix, AZ; ${ }^{4}$ Creighton University School of Medicine, Department of Anesthesiology, Omaha, NE; ${ }^{5}$ Louisiana State University Health Sciences Center, Department of Anesthesiology, New Orleans, LA

Author for correspondence: Ivan Urits, MD

Address: Beth Israel Deaconess Medical Center, William Arnold-Carol A. Warfield, MD Pain Center, 1 Brookline Place, Suite 105, Brookline, MA, 02445-7224

E-mail: Iurits@bidmc.harvard.edu ing a mechanical fall while walking. She described a sensation of sharp tugging and pressure in her low back with radiation down to her buttocks and posterior thighs bilaterally. Her motor exam revealed no deficits. Magnetic resonance imaging (MRI) demonstrated a large posterior extradural subfalcial fluid collection causing posterior thecal sac compression, isointense with cerebrospinal fluid (CSF) on T2-weighted imaging, concerning for CSF leak (Fig 1). Subsequent computed tomography (CT) guided aspiration of the collection provided relief of symptoms. A positive beta transferrin was reassuring for no cerebrospinal fluid accumulation. Her pain returned within 2 weeks of decompression and an additional MRI demonstrated return of her fluid collection (Fig 1b).

This case demonstrates the dramatic radiographic presentation of a post traumatic spinal seroma in the setting of prior fusion and instrumentation; moreover, its reoccurrence following imaging guided drainage. Seroma formation following spinal laminectomy and fusion is a rare though serious risk. Spinal seroma can develop after trauma or surgery and often requires surgical repair (3-5). Acutely, post-surgical seroma formation is well understood, and has been recognized to often be a result of recombinant human bone morphogenic protein use in graft placement (6). Delayed presentation of spinal seroma following surgical fusion, as in our patient, is seldom demonstrated and rather attributed to CSF collection secondary to dural leak (4). Spinal trauma following instrumentation may lead to hardware loosening and failure with instability and resultant fluid collection (3). As such, pain and radiculopathy following trauma in patients with a history of spinal instrumentation should warrant a low threshold for radiographic imaging. Though rare, delayed spinal seroma in post-surgical spinal fusion patients is an important complication which may require surgical management. 


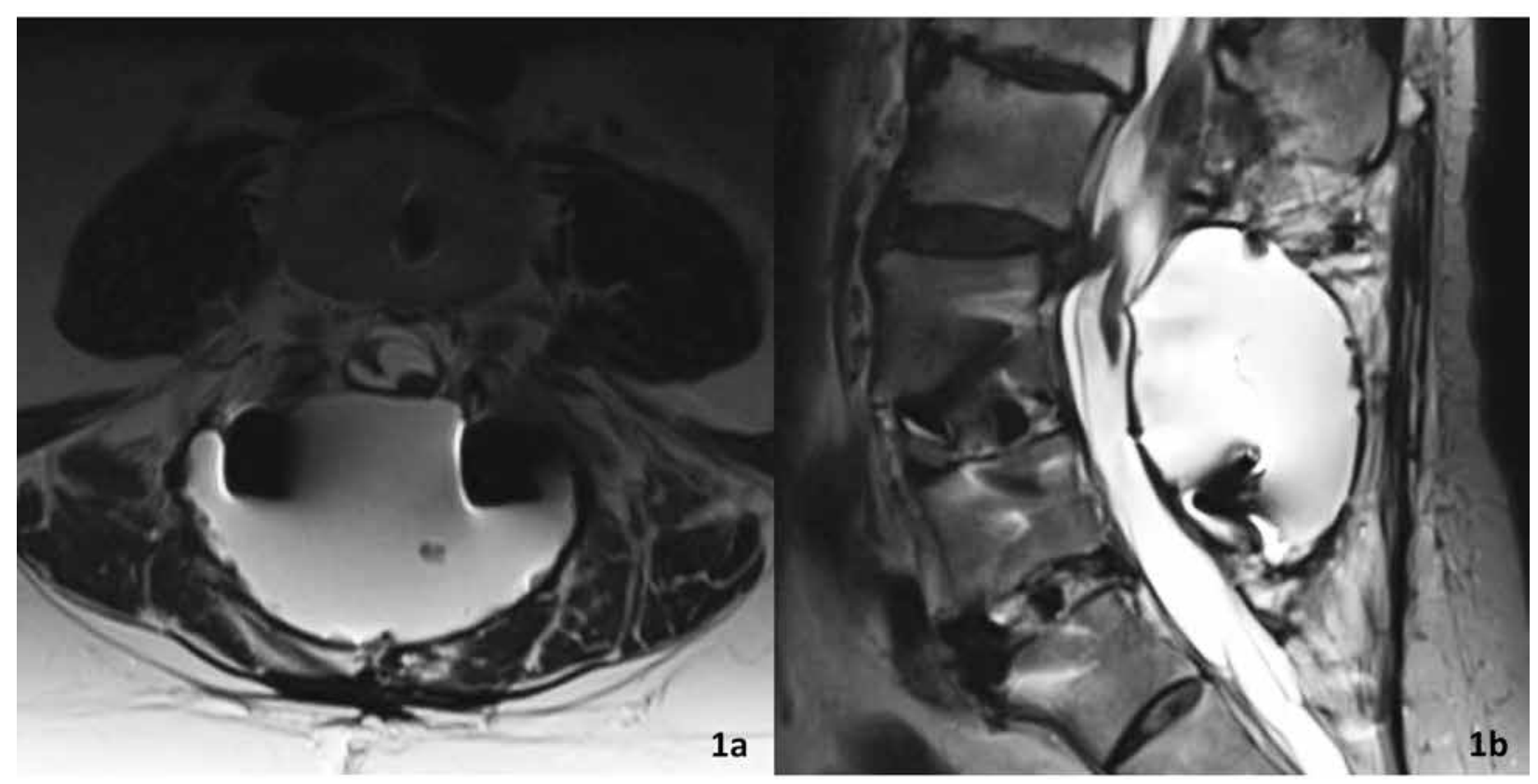

Fig. 1. T2 Axial (1a) and Sagittal MRI (1b) demonstrating a fluid collection, isointense on T2-weighted imaging with cerebrospinal fluid, within the surgical bed at the levels of L4-S1. Measurements of fluid collection: $7.1 \mathrm{~cm}$ in the transverse dimension, $4.4 \mathrm{~cm}$ in the AP dimension, and $6.9 \mathrm{~cm}$ in the craniocaudal dimension.

\section{REFERENCES}

1. Manchikanti L. Epidemiology of low back pain. Pain Physician 2000; 3:167-192.

2. Cortesi PA, Assietti R, Cuzzocrea F, et al. Epidemiologic and economic burden attributable to first spinal fusion surgery. Spine (Phila Pa 1976) 2017; 42:1398-1404.

3. Malhotra A, Kalra VB, Wu X, Grant R, Bronen RA, Abbed KM. Imaging of lumbar spinal surgery complications. Insights Imaging 2015; 6:579-590.

4. Khazim R, Dannawi Z, Spacey K, et al. Incidence and treatment of delayed symptoms of CSF leak following lumbar spinal surgery. Eur Spine ] 2015; 24:2069-2076.

5. Smorgick $\mathrm{Y}$, Baker KC, Herkowitz $\mathrm{H}$, et al. Predisposing factors for dural tear in patients undergoing lumbar spine surgery. J Neurosurg Spine 2015; 22:483-486.

6. Poorman GW, Jalai CM, Boniello A, Worley N, McClelland S, Passias PG. Bone morphogenetic protein in adult spinal deformity surgery: A meta-analysis. Eur Spine ] 2017; 26:2094-2102. 